\title{
Therapeutic experience with tramadol for opioid dependence in a patient with chronic low back pain: a case report
}

Marie Shigematsu-Locatelli, Takashi Kawano*, Tsuyoshi Koyama, Hideki Iwata, Atsushi Nishigaki, Bun Aoyama, Hiroki Tateiwa, Noriko Kitaoka and Masataka Yokoyama

\begin{abstract}
Background: Long-term opioid treatment for chronic non-cancer pain has become controversial, given the increasing prevalence of opioid dependence. However, there is little information on therapeutic strategies for this condition in Japanese patients. Here, we present a case of successful management of iatrogenic opioid dependence with tramadol in a patient with chronic low back pain.

Case presentation: A 68-year-old male suffering from intractable low back pain was referred to our pain clinic. He was previously treated in another hospital with transdermal fentanyl patches $6 \mathrm{mg} /$ day and fentanyl sublingual tablets $(100 \mu \mathrm{g}$ as required) for this condition. On the basis of medical examination, including a review of the patient's medical history, physical examination, X-ray, and his family statement, we diagnosed him with iatrogenic opioid dependence due to inadequate fentanyl use. Then, we developed a treatment plan consisting in fentanyl detoxification with a weak opioid, tramadol. At first, the use of fentanyl sublingual tablets was interrupted after obtaining informed consent. Then, we reduced the dose of transdermal fentanyl $1 \mathrm{mg}$ per 4-5 days replacing with oral sustained-release tramadol. The patient developed mild to moderate withdrawal symptoms during this period, which could be effectively managed by supportive treatments. The hospital psychiatry liaison team continuously provided the patient and his wife with information, counseling, and education regarding the treatment of opioid dependence. Throughout the detoxification process, his reported pain did not exacerbate, even slightly improved over time. The final prescription was sustained-release tramadol $300 \mathrm{mg} /$ day without fentanyl, and his activities of daily living drastically improved. However, unfortunately, he died due to an aortic dissection of stent-graft edge 65 days after surgery.
\end{abstract}

Conclusions: Our case highlighted that sustained-release tramadol could be effectively applied as a detoxification agent for iatrogenic opioid dependence in patients with chronic non-cancer pain.

Keywords: Opioid dependence, Tramadol, Chronic pain

\section{Background}

Opioid use disorders are well-known for leading to major health, social, and economic burden [1]. Specifically, iatrogenic opioid dependence or addiction is an emerging problem worldwide associated with increasing prescription rates. A recent meta-analysis shows an incidence of $4.7 \%$ within the patients that received opioid analgesic therapy [2]. In contrast, Japan has been

\footnotetext{
* Correspondence: takashika@kochi-u.ac.jp

Department of Anesthesiology and Intensive Care Medicine, Kochi Medical School, Kohasu, Oko-cho, Nankoku, Kochi 783-8505, Japan
}

considered to present one of the lowest incidences of inadequate opioid use due to two regulations, the narcotics control law and health care insurance system [3]. However, since 2010, opioid therapies including transdermal fentanyl patch were officially approved for chronic non-cancer pain. Therefore, although methamphetamine has been a more problematic substance than other drugs [4], there is ongoing concern regarding an increase in opioid use disorders. Based on such situation, the Japanese guideline for prescribing opioid analgesics for chronic non-cancer pain established the maximum 
daily dose as $120 \mathrm{mg}$ morphine milligram equivalent (MME) and emphasized the importance of preventing the development of opioid use disorders [5]. Meanwhile, there is a lack of information regarding the treatment strategies of iatrogenic opioid dependence in Japan. Here, we present a successful case of iatrogenic opioid dependence treatment using tramadol in a patient with intractable chronic low back pain.

\section{Case presentation}

A 68-year-old male suffering from intractable low back pain was referred to our pain clinic. He was previously treated in another hospital with transdermal fentanyl patches Fentos Tape $^{\oplus} 6 \mathrm{mg} /$ day and fentanyl sublingual tablets $\mathrm{Abstral}^{\circ} 100 \mu \mathrm{g}$ as required (usually 0-2 times/ day) for this condition. He was originally admitted to our hospital for a stent-graft insertion surgery for thoracic aorta aneurysm. Although the patient's postoperative pain was not predominant, his major complaint was regarding a worsening in continuous low back pain, leading to refuse rehabilitation. X-ray images showed an old L-4 lumbar compression fracture (Fig. 1), whereas we considered it may not sufficiently explain his severe pain. He also started to present behavioral problems confronting to the medical staff when requesting for

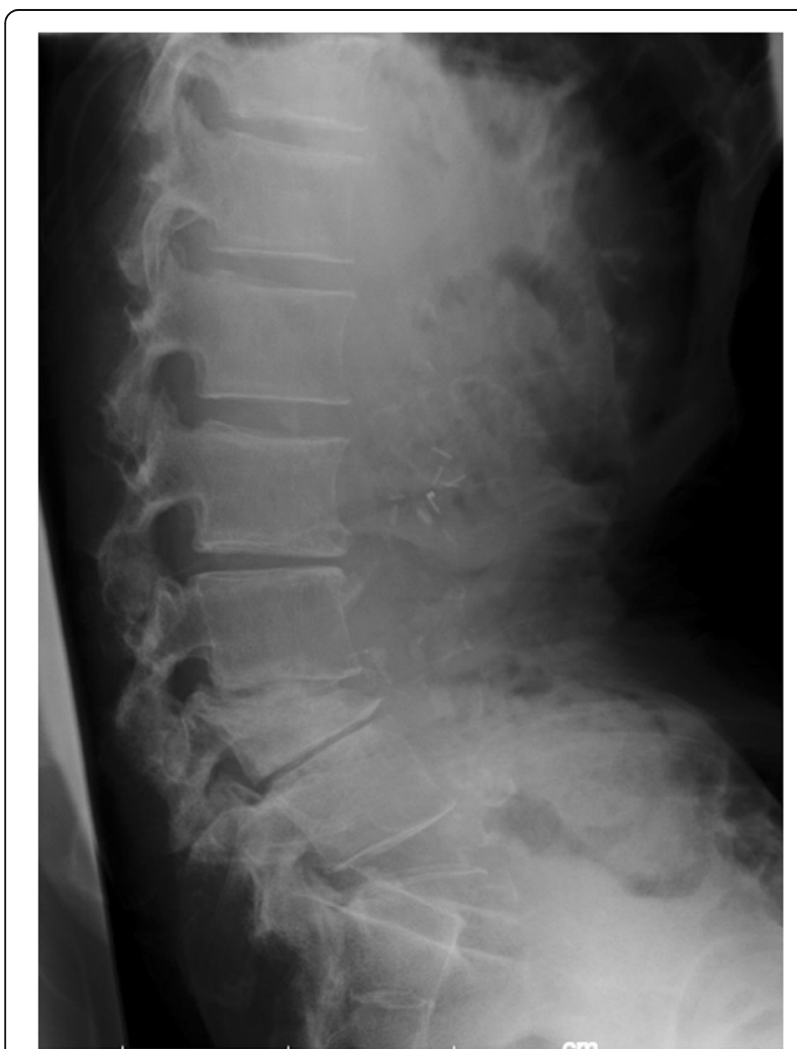

Fig. 1 A radiograph of lateral lumbar spine. Compression fracture of $\llcorner 4$ vertebral body is observed more opioids. After interviewing his wife, we determined that the patient may be using the fentanyl sublingual tablets not for pain control but for anxiety reduction. The resident physician and his attending nurse witnessed and recorded short myoclonus-like convulsions during the nighttime.

We suspected opioid-related disorders and investigated the patient's medical history for inadequate opioid use. His wife said that his personality used to be active, social, and industrious. However, 2 years before, he was diagnosed as terminal stage of cholangiocarcinoma by his family practitioner. This may be a misunderstanding, because his medical examinations including abdominal CT scans performed in our hospital revealed no sign of cancer. Erroneously believing that he had cancer, the patient became depressed, refusing further examinations, and his low back pain worsened. He also misunderstood that his low back pain was related to cancer metastasis. First-line analgesics, including acetaminophen, nonsteroidal anti-inflammatory drugs, and duloxetine, were prescribed, but none yielded satisfactory results. The patient only requested opioid prescription of transdermal fentanyl for his lumbar pain. His medical record showed that he had visited three different hospitals for his intractable pain symptom. During this period, the dose of transdermal fentanyl was gradually increased, as well as prescribed fentanyl sublingual tablet on an as-needed basis.

At the beginning, in order to rule out the possibility of opioid tolerance and pseudoaddiction, we switched opioids from fentanyl to equivalent dose of oral morphine (controlled-release formulations, P-guard ${ }^{\circ}$ ). However, no significant changes were observed in the pain symptom, as well as in the frequency of fentanyl sublingual tablet requirement. After that, the oral morphine was reverted to the transdermal fentanyl. Then, according to the International Statistical Classification of Diseases and Related Health Problems (ICD)-10 [6], we diagnosed the patient with opioid-related dependence (Additional file 1). In consequence, we developed a treatment plan that focused on fentanyl detoxification and replacement therapy with a weak opioid, tramadol. Before beginning treatment, we carefully explained to the patient and his wife about inappropriate fentanyl use and suspected opioid dependence. At first, in collaboration with the palliative care team, we informed them the importance of interrupting the use of fentanyl sublingual tablets and obtained their consent. Next, we reduced the dose of transdermal fentanyl $1 \mathrm{mg}$ per 4-5 days replacing with oral tramadol (Onetram ${ }^{\circ}$, sustained-release tablet) (Fig. 2). During this treatment period, the patient developed mild to moderate withdrawal symptoms, such as abdominal pain with diarrhea, insomnia, and anxiety. These symptoms could be effectively managed by 


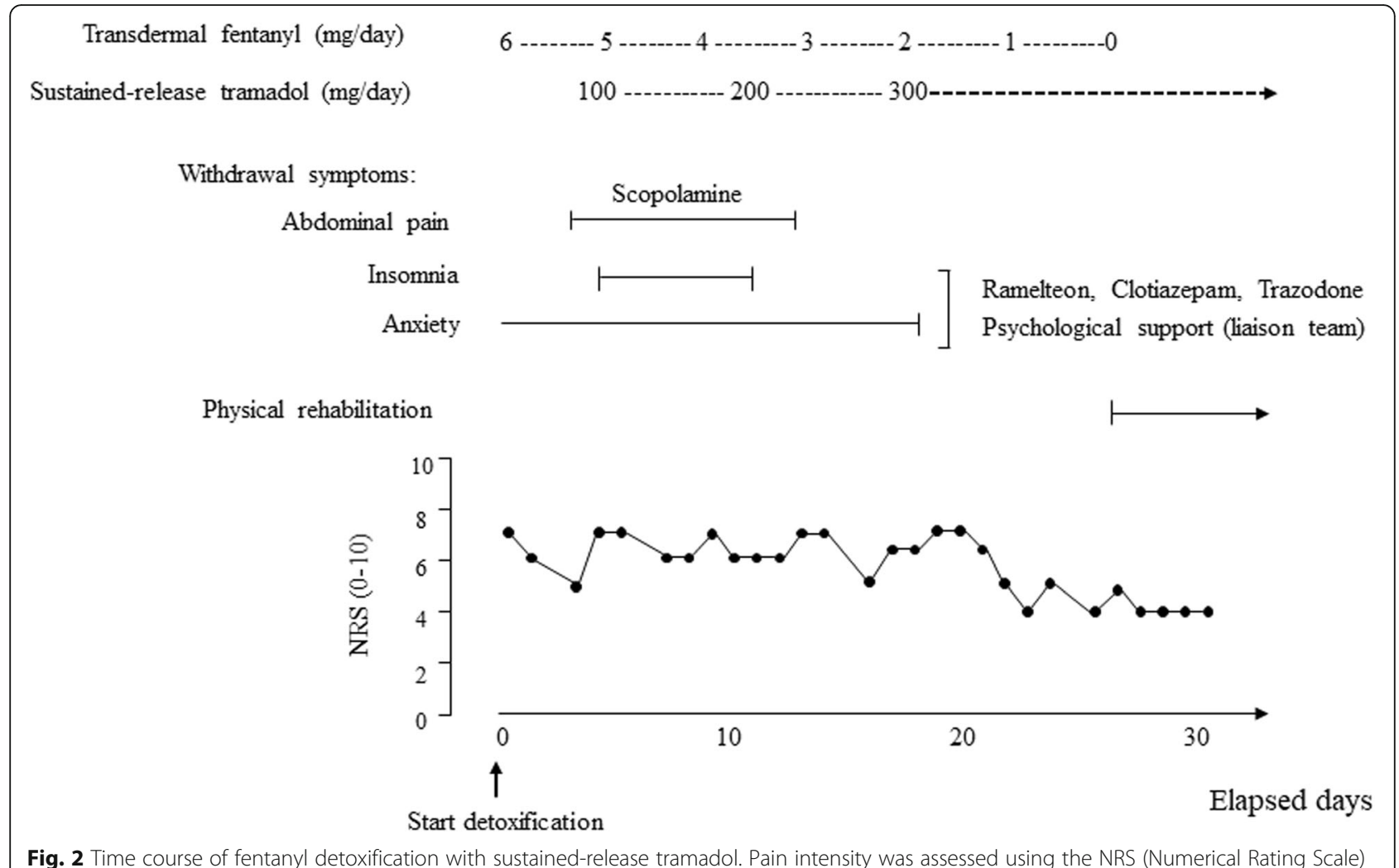

Fig. 2 Time course of fentanyl detoxification with sustained-release tramadol. Pain intensity was assessed using the NRS (Numerical Rating Scale)

supportive treatment, i.e., abdominal pain was treated by scopolamine butyl bromide, and the insomnia and anxiety were controlled with ramelteon $8 \mathrm{mg}$, clotiazepam 5 $\mathrm{mg}$, and trazodone hydrochloride $25 \mathrm{mg}$. The patient did not complain about pain exacerbation throughout the treatment (Fig. 2), and no additional analgesic was required. The hospital psychiatry liaison team continued to provide the patient and his wife with information, counseling, and education regarding the treatment of opioid dependence. After the completion of our treatment program, his activities of daily living drastically improved, and the patient could start rehabilitation. The final prescription was tramadol $300 \mathrm{mg} /$ day without fentanyl, which continued to be prescribed for his lower back pain. However, unfortunately, he died due to an aortic dissection of stent-graft edge 65 days after surgery.

\section{Discussion}

We described a case of iatrogenic opioid dependence induced by inadequate fentanyl use for chronic noncancer pain. The patient was successfully detoxified by sustained-released tramadol without severe withdrawal symptoms.

Iatrogenic opioid dependence is a serious adverse effect associated with long-term opioid therapy for chronic non-cancer pain $[1,7]$. In order to prevent its development, it is critically important for the physician to prescribe opioids appropriately. Specifically, since highdose opioid prescription is one of the major risk factors, the Japanese clinical practice guideline recommends the maximum daily opioid dose as $120 \mathrm{mg}$ of MME [5]. In addition, sublingual formulation of fentanyl can be used to achieve a rapid onset of action, which may enhance abuse potential [8]. Accordingly, it is only indicated for breakthrough pain in opioid-treated cancer patients. In our case, however, the patient treated his chronic low back pain with transdermal fentanyl $6 \mathrm{mg}$ (180 mg of MME) in combination with sublingual fentanyl tablets. This inappropriate opioid use may be a consequence of complicated circumstances, such as patient misinterpretation, patient-physician miscommunication, inadequate physician attitudes towards opioids, and lack of drug monitoring systems.

Inadequate pain management led to drug-seeking behaviors that resemble those seen with opioid addiction. This phenomenon is referred to as pseudoaddiction [9, 10]. In clinical settings, pseudoaddiction can be distinguished from opioid dependence in that the behaviors disappear after appropriate pain treatment. On the other hand, opioid tolerance develops with repeated opioid exposure, resulting in a decrease in the analgesic effect [10, 11]. The development of opioid tolerance has been reported to lead to an increase in opioid consumption and the risk of addiction. Opioid switching or rotation is the 
process of substituting one opioid for another for improvement of opioid responsiveness [11]. In our case, despite an opioid switching from transdermal fentanyl to equi-analgesic dose of oral morphine, no significant pain relief was observed. Furthermore, his pain intensity did not exacerbate during fentanyl detoxification, even presented a slight improvement over time (Fig. 2), indicating that the occurrence of pseudoaddiction could be excluded.

A crucial first step in the treatment of opioid dependency is detoxification [12, 13]. Opioid detoxification is the supervised withdrawal from causative opioid to minimize withdrawal symptoms. Currently, two types of Food and Drug Administration (FDA)-approved medications, oral methadone and sublingual buprenorphine, are widely used as the standard agents for medical opioid detoxification [12, 13]. Methadone is a full opioid agonist with long half-life and high affinity at the $\mu$-opioid receptor, which can ameliorate the euphoria effects and withdrawal symptoms [14]. Buprenorphine is a partial opioid agonist, which makes it an advantageous alternative to methadone due to its relatively safe profile [15]. Evidence shows that both medications help patients to reach a stabilized physical condition, which then allows them to successfully go through counseling and rehabilitation programs [12-15]. However, they are not currently prescribed in Japan, i.e., oral methadone is only indicated for the treatment of cancer pain, and sublingual formulation of buprenorphine has not been available yet.

Tramadol is a centrally acting analgesic for treatment of moderate to severe pain in both cancer and noncancer patients [16]. Since it binds to $\mu$-opioid receptors, tramadol could relieve opioid withdrawal symptoms [17]. In fact, a previous clinical trial showed that oral treatment of immediate-release tramadol (200 or 400 $\mathrm{mg}$ ) reduces opioid withdrawal in morphine-maintained adults [18]. Another report further demonstrated that the extended-release tramadol of therapeutic dose $(200$ mg daily) was more efficacious than placebo and supratherapeutic dose (600 mg daily) in treating withdrawal in opioid dependence patients [19]. In contrast, a recent randomized controlled trial showed that higher doses of extended-release tramadol (up to $600 \mathrm{mg}$ daily) suppressed opioid withdrawal symptom comparably to buprenorphine [20]. Therefore, relatively small dose regimen (up to $300 \mathrm{mg}$ daily) may explain why some withdrawal symptoms were developed in our case.

On the other hand, the affinity of tramadol for the $\mu$ opioid receptor is very low, i.e., 6000 -fold less than that of morphine [16]. It may be associated with less incidence of side effects, including lower rates of respiratory depression and reduced abuse liability, compared to other $\mu$-opioid analgesics. Specifically, compared with immediate-release formulations, sustained-release tramadol could provide a more controlled, safe, and prolonged analgesic effect, minimizing adverse effects associated with uncontrolled drugs peak plasma levels [21]. Furthermore, tramadol inhibits the neuronal re-uptake of noradrenaline and serotonin, which contributes to better analgesic effects for chronic pain via activation of descending inhibitory pathways $[16,21]$. These pharmacological profiles, along with our case, suggest that the medication with sustained-release tramadol could be an effective alternative approach for supervised opioid detoxification in patients with chronic non-cancer pain. Nevertheless, several important questions remain such as long-term effectiveness and optimal dose regimen. In addition, a Cochrane database systemic review published in 2018 reported that there were insufficient data to determine whether tramadol differs from methadone in alleviating withdrawal symptoms [17]. Therefore, further clinical studies regarding our hypothesis are necessary and warranted.

\section{Conclusion}

Although detoxification is one essential component of an effective treatment for opioid dependence, none of the FDA-approved medications are currently available in Japan. Our case highlighted that sustained-release tramadol could be a safe alternative detoxification agent for opioid dependence in patients with chronic non-cancer pain.

\section{Supplementary information}

Supplementary information accompanies this paper at https://doi.org/10. 1186/s40981-019-0289-z

Additional file 1. Diagnosis of opioid-related dependence. (DOCX 13 $\mathrm{kb})$

\section{Abbreviations}

FDA: Food and Drug Administration; ICD: International Statistical Classification of Diseases and Related Health Problems; MME: Morphine milligram equivalent

\section{Acknowledgements \\ Not applicable. \\ Authors' contributions \\ All authors contributed to writing the manuscript and approved the final version of the manuscript for submission. \\ Funding \\ No funding. \\ Availability of data and materials \\ The data are not available for public access because of patient privacy concerns, but are available from the corresponding author on reasonable request.}

Ethics approval and consent to participate Not applicable. 


\section{Consent for publication}

Written informed consent was obtained from the patient for publication of this case report and any accompanying images.

\section{Competing interests}

The authors declare that they have no competing interests.

Received: 13 September 2019 Accepted: 1 October 2019

Published online: 30 October 2019

\section{References}

1. Speed TJ, Parekh V, Coe W, Antoine D. Comorbid chronic pain and opioid use disorder: literature review and potential treatment innovations. Int Rev Psychiatry. 2018;30:136-46.

2. Higgins $C$, Smith BH, Matthews K. Incidence of iatrogenic opioid dependence or abuse in patients with pain who were exposed to opioid analgesic therapy: a systematic review and meta-analysis. Br J Anaesth. 2018;120:1335-44.

3. Onishi E, Kobayashi T, Dexter E, Marino M, Maeno T, Deyo RA. Comparison of opioid prescribing patterns in the United States and Japan: primary care physicians' attitudes and perceptions. J Am Board Fam Med. 2017:30:24854.

4. Wada K, Funada M, Shimane T. Current status of substance abuse and HIV infection in Japan. J Food Drug Anal. 2013;21:S33-6.

5. Committee for the Guidelines for Prescribing Opioid Analgesics for Chronic Noncancer Pain of JSPC. Guidelines for prescribing opioid analgesics for chronic noncancer pain. Tokyo: Sinko Trading Co. Ltd; 2012.

6. World Health Organization. International statistical classification of diseases and related health problems. $10^{\text {th }}$ Revision. Geneva: World Health Organization; 1992.

7. Beauchamp GA, Winstanley EL, Ryan SA, Lyons MS. Moving beyond misuse and diversion: the urgent need to consider the role of iatrogenic addiction in the current opioid epidemic. Am J Public Health. 2014;104:2023-9.

8. Gasior M, Bond M, Malamut R. Routes of abuse of prescription opioid analgesics: a review and assessment of the potential impact of abusedeterrent formulations. Postgrad Med. 2016;128:85-96.

9. Lusher J, Elander J, Bevan D, Telfer P, Burton B. Analgesic addiction and pseudoaddiction in painful chronic illness. Clin J Pain. 2006;22:316-24.

10. Morrone LA, Scuteri D, Rombolà L, Mizoguchi H, Bagetta G. Opioids resistance in chronic pain management. Curr Neuropharmacol. 2017;15:44456.

11. Vissers KC, Besse K, Hans G, Devulder J, Morlion B. Opioid rotation in the management of chronic pain: where is the evidence? Pain Pract. 2010;10: 85-93.

12. Kleber HD. Pharmacologic treatments for opioid dependence: detoxification and maintenance options. Dialogues Clin Neurosci. 2007;9:455-70.

13. Stotts AL, Dodrill CL, Kosten TR. Opioid dependence treatment: options in pharmacotherapy. Expert Opin Pharmacother. 2009;10:1727-40.

14. Ali S, Tahir B, Jabeen S, Malik M. Methadone treatment of opiate addiction: a systematic review of comparative studies. Innov Clin Neurosci. 2017;14:819

15. Ling W. Buprenorphine for opioid dependence. Expert Rev Neurother. 2009; 9:609-16.

16. Grond S, Sablotzki A. Clinical pharmacology of tramadol. Clin Pharmacokinet. 2004;43:879-923.

17. Rahimi-Movaghar A, Gholami J, Amato L, Hoseinie L, Yousefi-Nooraie R, Amin-Esmaeili M. Pharmacological therapies for management of opium withdrawal. Cochrane Database Syst Rev. 2018:6:CD007522.

18. Lofwall MR, Walsh SL, Bigelow GE, Strain EC. Modest opioid withdrawal suppression efficacy of oral tramadol in humans. Psychopharmacology. 2007;194:381-93.

19. Lofwall MR, Babalonis S, Nuzzo PA, Siegel A, Campbell C, Walsh SL. Efficacy of extended-release tramadol for treatment of prescription opioid withdrawal: a two-phase randomized controlled trial. Drug Alcohol Depend. 2013:133:188-97.

20. Dunn KE, Tompkins DA, Bigelow GE, Strain EC. Efficacy of tramadol extended-release for opioid withdrawal: a randomized clinical trial. JAMA Psychiatry. 2017;74:885-93.

21. Mongin G. Tramadol extended-release formulations in the management of pain due to osteoarthritis. Expert Rev Neurother. 2007;7:1775-84.

\section{Publisher's Note}

Springer Nature remains neutral with regard to jurisdictional claims in published maps and institutional affiliations.

\section{Submit your manuscript to a SpringerOpen ${ }^{\circ}$ journal and benefit from:}

- Convenient online submission

- Rigorous peer review

- Open access: articles freely available online

- High visibility within the field

- Retaining the copyright to your article

Submit your next manuscript at $\boldsymbol{\nabla}$ springeropen.com 